\title{
A Product Formula and Its Application to the Schrödinger Equation
}

By

\author{
Takashi ICHINOSE*
}

\begin{abstract}
A product formula is given which represents the unitary group for the form sum of a pair of nonnegative selfadjoint operators in a Hilbert space.
\end{abstract}

\section{Introduction}

The form sum of nonnegative selfadjoint operators $A$ and $B$ in a Hilbert space $\mathscr{H}$, denoted by $A+B$, is the nonnegative selfadjoint operator associated with the closed nonnegative quadratic form

$$
u \longmapsto\left\|A^{1 / 2} u\right\|^{2}+\left\|B^{1 / 2} u\right\|^{2}
$$

defined on $D\left[A^{1 / 2}\right] \cap D\left[B^{1 / 2}\right]$, the intersection of the form domains for $A$ and $B$. Here it is assumed for simplicity that this intersection is dense in $\mathscr{H}$.

The aim of the present paper is to prove the following product formula representing the unitary group for $A+B$ :

$$
\begin{gathered}
\mathrm{s}-\lim _{n \rightarrow \infty}\left\{\left[e^{i \varepsilon t A / n} E_{A}([0, n \delta /|t|])+e^{i \varepsilon t a / n} E_{A}((n \delta /|t|, \infty))\right]\right. \\
\left.\cdot\left[e^{i \varepsilon t B / n} E_{B}([0, n \delta /|t|])+e^{i \varepsilon t b / n} E_{B}((n \delta /|t|, \infty))\right]\right\}^{n} \\
=e^{i \varepsilon t(A \dot{+} B)}, \quad-\infty<t<\infty, \varepsilon= \pm 1 .
\end{gathered}
$$

Here $\left\{E_{A}(\lambda)\right\}$ and $\left\{E_{B}(\lambda)\right\}$ are the spectral families associated with $A$ and $B$ respectively. $a \geq 0, b \geq 0$ and $0<\delta<\pi / 2$ are arbitrary fixed constants.

Kato [8] and Kato-Masuda [9] have proved the Trotter product formula of the selfadjoint case which represents the selfadjoint contraction semigroup for $A+B$ :

$$
\mathrm{S}-\lim _{n \rightarrow \infty}\left\{e^{-t A / n} e^{-t B / n}\right\}^{n}=e^{-t(A \dot{+} B)}, \quad t \geq 0 .
$$

Communicated by H. Araki, January 12, 1979.

* Department of Mathematics, Hokkaido University. 
The proof makes use of the Chernoff theorem [1] and the techniques employed in Kato [8]. Although the formula (0.1) deviates from the Trotter product formula of the unitary case, i.e. the formula $(0.1)$ with $\delta=\infty$, the approximation procedure involved may be of some use; the contributions from $A$ and $B$ are obtained first by replacing their spectral values outside a finite interval $[0, n \delta /|t|]$ with fixed constants $a \geq 0$ and $b \geq 0$ and then by taking a limit $n \rightarrow \infty$.

The formula (0.1) is applied to an integral representation of the solution of the Schrödinger equation

$$
i \frac{\partial}{\partial t} \psi(t, x)=\left[-\frac{1}{2} \Delta+V(x)\right] \psi(t, x), \quad t>0,
$$

in $\boldsymbol{R}^{m}$ with initial condition $\psi(0, x)=f(x)$. Here $V(x)$ is a real-valued measurable function bounded from below. A characteristic feature of the present result is the truncation process of the operators $-(1 / 2) \Delta$ and $V$ in obtaining an integral representation of the solution of $(0.3)$.

Section 1 is devoted to statement of the main results. The proof is given in Section 2. Section 3 is concerned with a general class of product formulas including (0.1).

For related problems see Chernoff [1], Chorin et al. [2], Faris [3], Kato [7], [8], Kato-Masuda [9] and Nelson [11].

\section{§1. The Main Results}

\subsection{A product formula.}

Let $A$ and $B$ be selfadjoint operators in a Hilbert space $\mathscr{H}$ with domains $D[A]$ and $D[B]$ and with spectral families $\left\{E_{A}(\lambda)\right\}$ and $\left\{E_{B}(\lambda)\right\}$, respectively. Assume that $A$ and $B$ are semibounded, in fact, without losing any generality, bounded from below with lower bounds $\gamma_{A}$ and $\gamma_{B}$; they need not be the greatest ones. $A$ and $B$ have the spectral representations

$$
A=\int_{-\infty}^{\infty} \lambda d E_{A}(\lambda), \quad B=\int_{-\infty}^{\infty} \lambda d E_{B}(\lambda)
$$

Let $\mathscr{H}_{0}$ be the closure of

$$
D\left[\left(A-\gamma_{A}\right)^{1 / 2}\right] \cap D\left[\left(B-\gamma_{B}\right)^{1 / 2}\right] \text {. }
$$

$\mathscr{H}_{0}$ is a Hilbert space as a closed subspace of $\mathscr{H}$. Let $P$ be the orthogonal 
projection of $\mathscr{H}$ onto $\mathscr{H}_{0}$. We denote by $A+B$ the form sum of $A$ and $B$, which is the selfadjoint operator in $\mathscr{H}_{0}$ bounded from below associated with the closed quadratic form bounded from below

$$
u \longmapsto \int_{-\infty}^{\infty} \lambda d\left\|E_{A}(\lambda) u\right\|^{2}+\int_{-\infty}^{\infty} \lambda d\left\|E_{B}(\lambda) u\right\|^{2}
$$

defined on the subspace (1.1) of $\mathscr{H}_{0}$ (see Kato [5], Reed-Simon [12]). The form sum $A+B$ coincides with the closure of the operator sum $A+B$ with domain $D[A+B]=D[A] \cap D[B]$, if $A+B$ is essentially selfadjoint in $\mathscr{H}$.

The main result is the following theorem.

Theorem 1. For $\varepsilon= \pm 1$ and for $f \in \mathscr{H}_{0}=P \mathscr{H}$,

$$
\begin{aligned}
& \left\{\left[e^{i \varepsilon t A / n} E_{A}\left(\left[\gamma_{A}, \gamma_{A}+n \delta / t\right]\right)+e^{i \varepsilon t a / n} E_{A}\left(\left(\gamma_{A}+n \delta / t, \infty\right)\right)\right]\right. \\
& \left.\quad \cdot\left[e^{i \varepsilon t B / n} E_{B}\left(\left[\gamma_{B}, \gamma_{B}+n \delta / t\right]\right)+e^{i \varepsilon t b / n} E_{B}\left(\left(\gamma_{B}+n \delta / t, \infty\right)\right)\right]\right\}^{n} f \\
& \quad \rightarrow e^{i \varepsilon t\left(A^{+}+B\right)} f, \quad n \rightarrow \infty,
\end{aligned}
$$

uniformly on bounded intervals in $t \geq 0 .{ }^{1)} \quad$ Here $a, b$ and $\delta$ are arbitrary fixed real constants satisfying

$$
a \geq \gamma_{A}, \quad b \geq \gamma_{B}, \quad 0<\delta<\pi / 2 .
$$

\section{Remarks}

1. The sign $\varepsilon$ in the formula (1.3) has a double purpose. It replaces $t$ with $-t$ and semibounded operators from below with those from above. Another formulation of Theorem 1 is the following: For $\varepsilon= \pm 1$ and for $f \in \mathscr{H}_{0}=P \mathscr{H},(1.3)$ with $n \delta /|t|$ in place of $n \delta / t$ is valid, uniformly on bounded intervals in $-\infty<t<\infty$. In this case, $\varepsilon$ is used only for the second purpose.

2. The problem is open whether Theorem 1 is valid for $\delta$ with $\pi / 2 \leq \delta \leq \infty$. However, whenever $A+B$ is essentially selfadjoint in $\mathscr{H}$, the formula (1.3) with $\delta=\infty$ and $\mathscr{H}_{0}=\mathscr{H}$ and with the closure of $A+B$ in place of $A+B$, i.e. the Trotter product formula of the unitary case holds, even if $A$ and $B$ are not necessarily semibounded.

3. It is not clear whether $a$ and $b$ can be replaced by $\gamma_{A}+n \delta / t$ and $\gamma_{B}+n \delta / t$, respectively.

4. Suppose that $\mathscr{H}_{0}=\mathscr{H}$ and $A$ is nonnegative. In a case where $B$ may not be semibounded, Faris [3] has given another product formula, but with an assumption that $B$ is relatively form-bounded with respect to $A$ with relative

1) For $t=0$ the upper member of (1.3) is supposed to be $f$. 
bound $<1$ (cf. Simon [13, pp. 51-53]). This case is not covered by Theorem 1 , the proof of which greatly depends upon semiboundedness or nonnegativity of both $A$ and $B$. We note that the Faris formula contains two limits, however.

5. The selfadjoint analogue to Theorem 1 is valid for $0<\delta<\infty$; for $f \in \mathscr{H}$,

$$
\begin{aligned}
& \left\{\left[e^{-t A / n} E_{A}\left(\left[\gamma_{A}, \gamma_{A}+n \delta / t\right]\right)+^{-t a / n} E_{A}\left(\left(\gamma_{A}+n \delta / t, \infty\right)\right)\right]\right. \\
& \left.\quad \cdot\left[e^{-t B / n} E_{B}\left(\left[\gamma_{B}, \gamma_{B}+n \delta / t\right]\right)+e^{-t b / n} E_{B}\left(\left(\gamma_{B}+n \delta / t, \infty\right)\right)\right]\right\}^{n} f \\
& \quad \rightarrow e^{-t(A+\dot{B})} P f, \quad n \rightarrow \infty,
\end{aligned}
$$

uniformly on bounded intervals in $t>0$, and on bounded intervals in $t \geq 0$ when $f \in \mathscr{H}_{0}$. Here $a \geq \gamma_{A}$ and $b \geq \gamma_{B}$. This is contained in the result of Kato [7].

6. In the case $\mathscr{H}_{0} \neq \mathscr{H}$, the limit of the left-hand side of (1.3) cannot exists for every $f \in \mathscr{H}$ and for every $t \geq 0$. In fact, if it exists, by [1, Corollary 2.5.4] it should have the form $e^{i \varepsilon t C} f$ for some selfadjoint operator $C$ in $\mathscr{H}$, so that the limit of (1.4) in Remark 5 above should be $e^{-t C} f$ because the left-hand side of (1.3) is holomorphic in the upper half plane $\operatorname{Im} \varepsilon>0$. This is only possible if $P=1$ or $\mathscr{H}_{0}=\mathscr{H}$.

\subsection{An integral representation of the solution of the Schrödinger equation.}

We apply Theorem 1 to the Schrödinger equation (0.3) with potential a real-valued measurable function $V(x)$ in $\boldsymbol{R}^{m}$. When the operator sum $-(1 / 2) \Delta$ $+V$ is essentially selfadjoint in $L^{2}\left(\boldsymbol{R}^{m}\right)$, Nelson [11] has used the Trotter product formula of the unitary case to illustrate the Feynman path integral representation of the solution of (0.3).

We are interested in the same problem in the case $-(1 / 2) \Delta+V$ may not necessarily be essentially selfadjoint. We assume that $V(x)$ is bounded from below, $V(x) \geq \gamma$, and that

$$
\begin{aligned}
& D\left[\left(-\frac{1}{2} \Delta\right)^{1 / 2}\right] \cap D\left[|V|^{1 / 2}\right] \\
& \quad=\left\{\phi \in W^{1}\left(\boldsymbol{R}^{m}\right) ;|V|^{1 / 2} \phi \in L^{2}\left(\boldsymbol{R}^{m}\right)\right\}
\end{aligned}
$$

is dense in $L^{2}\left(\boldsymbol{R}^{m}\right)$. Let $H$ be the selfadjoint operator associated with the closed quadratic form bounded from below

$$
\phi \longmapsto \frac{1}{2} \int_{\boldsymbol{R}^{m}}|\nabla \phi(x)|^{2} d x+\int_{\boldsymbol{R}^{m}} V(x)|\phi(x)|^{2} d x
$$

defined on the subspace (1.5) of $L^{2}\left(\boldsymbol{R}^{m}\right)$. 
If $V$ is in $L_{\text {loc }}^{2}\left(\boldsymbol{R}^{m}\right)$ and bounded from below, then, by a result of Kato [6] (see Reed-Simon [12, Theorem X.28]), $-(1 / 2) \Delta+V$ is always essentially selfadjoint. However, there are examples of $V(x)$ for which $D[-(1 / 2) \Delta] \cap D[V]$ $=\{0\}$ but $(1.5)$ contains sufficiently many elements of $L^{2}\left(\boldsymbol{R}^{m}\right)$ (see Simon $[13$, in particular, I.6. Examples] and Chernoff $[1, \S 5])$.

The following result is a direct consequence of Theorem 1. The phase space path integral may come to mind.

Theorem 2. For $t>0$ and for $f \in L^{2}\left(\boldsymbol{R}^{m}\right)$,

$$
\begin{aligned}
\left(e^{-i t H} f\right)(x) & \\
=\lim _{n \rightarrow \infty} & \int_{\boldsymbol{R}^{2 m}} \cdots \int_{\boldsymbol{R}^{2 m}} \exp \left\{i \left[\left(x-x_{1}\right) \xi_{1}+\left(x_{1}-x_{2}\right) \xi_{2}\right.\right. \\
& \left.\left.+\cdots+\left(x_{n-1}-x_{n}\right) \xi_{n}\right]\right\} \\
& \cdot \prod_{j=1}^{n}\left\{\left[\Xi\left(t / n ; \xi_{j}\right) \exp \left(-i t \xi_{j}^{2} / 2 n\right)+\Xi^{\prime}\left(t / n ; \xi_{j}\right) \exp (-i t a / n)\right]\right. \\
& \left.\cdot\left[X\left(t / n ; x_{j}\right) \exp \left(-i t V\left(x_{j}\right) / n\right)+X^{\prime}\left(t / n ; x_{j}\right) \exp (-i t b / n)\right]\right\} \\
& \cdot f\left(x_{n}\right) \prod_{j=1}^{n}(2 \pi)^{-m} d \xi_{j} d x_{j} .
\end{aligned}
$$

Here $\Xi, \Xi^{\prime}, X$ and $X^{\prime}$ are functions in $\boldsymbol{R}^{m}$ defined by

$$
\begin{aligned}
& \Xi(t ; \xi)=\left\{\begin{array}{ll}
1, & \xi^{2} / 2 \leq \delta / t, \\
0, & \xi^{2} / 2>\delta / t,
\end{array} \quad X(t ; x)= \begin{cases}1, & \gamma \leq V(x) \leq \delta / t, \\
0, & V(x)>\delta / t,\end{cases} \right. \\
& \Xi^{\prime}(t ; \xi)=1-\Xi(t ; \xi),
\end{aligned}
$$

and $a \geq 0, b \geq \gamma$ and $0<\delta<\pi / 2$ are arbitrary fixed real constants. The limit is taken in the sense of $L^{2}\left(\boldsymbol{R}^{m}\right)$. The $n$-ple integral is a repeated one, and each integral is taken in the sense

$$
\int_{\boldsymbol{R}^{2 m}}(2 \pi)^{-m} d \xi_{j} d x_{j}=\lim _{r \rightarrow \infty} \int_{\left|\xi_{j}\right| \leq r,\left|x_{j}\right| \leq r}(2 \pi)^{-m} d \xi_{j} d x_{j}, 1 \leq j \leq n
$$

the limit taken in the sense of $L^{2}\left(\boldsymbol{R}^{m}\right)$ with respect to the variable $x_{j-1}$, where $x_{0}=x$.

Remark. Occurrence of the cutoff functions $\Xi\left(t / n ; \xi_{j}\right), \Xi^{\prime}\left(t / n ; \xi_{j}\right)$ and $X\left(t / n ; x_{j}\right), X^{\prime}\left(t / n ; x_{j}\right), 1 \leq j \leq n$, makes the formula (1.6) deviate from the formal approximation formula (i.e. the formula(1.6) with $\Xi=X \equiv 1$ and $\Xi^{\prime}=X^{\prime} \equiv 0$ ) to the phase space path integral (e.g. Garrod [4], Mizrahi [10]). However, it is worth noticing that (1.6) does involve an interesting approximation procedure in which the potential $V(x)$ is cut down to a fixed constant $b \geq \gamma$ outside the region $\gamma \leq$ 
$V(x) \leq n \delta / t$ in the configuration space, and the free Hamiltonian $-(1 / 2) \Delta$, in the momentum representation, cut down to a fixed constant $a \geq 0$ outside the region $\xi^{2} / 2 \leq n \delta / t$. For another choice of the cutoff functions see Remark 2 to Theorem 3 in Section 3.

\section{$\S 2$. The Proof}

In Section 2.1 we prove Theorem 1, using the techniques in Kato [8]. The proof needs some lemmas, whose proofs will be given in Section 2.2.

\subsection{Proof of Theorem 1 .}

We shall only treat the case $\varepsilon=-1$. It is not difficult to see that the general case can be reduced to the case $\gamma_{A}=\gamma_{B}=0$ by considering $A-\gamma_{A}$ and $B-\gamma_{B}$ instead of $A$ and $B$. Thus we assume that both $A$ and $B$ are nonnegative selfadjoint with $\gamma_{A}=\gamma_{B}=0$. Set $C=A+B . \quad C$ is a nonnegative selfadjoint operator in $\mathscr{H}_{0}, C: D[C] \subset \mathscr{H}_{0} \rightarrow \mathscr{H}_{0}$, such that

$$
\begin{aligned}
& D\left[C^{1 / 2}\right]=D\left[A^{1 / 2}\right] \cap D\left[B^{1 / 2}\right], \\
& \left\|C^{1 / 2} u\right\|^{2}=\left\|A^{1 / 2} u\right\|^{2}+\left\|B^{1 / 2} u\right\|^{2}, \quad u \in D\left[C^{1 / 2}\right] .
\end{aligned}
$$

Let $a \geq 0, b \geq 0$ and $0<\delta<\pi / 2$ be fixed. Define for $t \geq 0$

$$
\begin{aligned}
F(0)= & 1, \\
F(t)= & {\left[e^{-i t A} E_{A}([0, \delta / t])+e^{-i t a} E_{A}((\delta / t, \infty))\right] } \\
& \cdot\left[e^{-i t B} E_{B}([0, \delta / t])+e^{-i t b} E_{B}((\delta / t, \infty))\right], \quad t>0 .
\end{aligned}
$$

Then $\{F(t)\}_{t \geq 0}$ is a family of linear contractions in $\mathscr{H}$. We must show that, for $f \in \mathscr{H}_{0}=P \mathscr{H}$,

$$
F(t / n)^{n} f \rightarrow e^{-i t C} f, \quad n \rightarrow \infty,
$$

uniformly on bounded intervals in $t \geq 0$.

We apply now the Chernoff theorem [1, Theorem 1.1], which can be modified to fit the present case. (For this context we refer to Kato [8].) We note also the fact [5, IX-Theorem 2.16]. Thus to prove (2.3) we have only to show that

$$
\mathrm{s}-\lim _{t \downarrow 0}\left[1+i-t^{-1}(F(t)-1)\right]^{-1}=(1+i+i C)^{-1} P .
$$

Let us introduce a few notations. Set 
(2.5)

$$
\begin{aligned}
A(t) & =(i t)^{-1}\left\{1-\left[e^{-i t A} E_{A}([0, \delta / t])+e^{-i t a} E_{A}((\delta / t, \infty))\right]\right\} \\
& =\left(\frac{1-\exp (-i t A)}{i t}\right) E_{A}([0, \delta / t])+\left(\frac{1-\exp (-i t a)}{i t}\right) E_{A}((\delta / t, \infty)) \\
B(t) & =(i t)^{-1}\left\{1-\left[e^{-i t B} E_{B}([0, \delta / t])+e^{-i t b} E_{B}((\delta / t, \infty))\right]\right\} \\
& =\left(\frac{1-\exp (-i t B)}{i t}\right) E_{B}([0, \delta / t])+\left(\frac{1-\exp (-i t b)}{i t}\right) E_{B}((\delta / t, \infty))
\end{aligned}
$$

For $i A(t)$ we denote by $|A(t)|$ the absolute value, and by $G_{A}(t)$ and $H_{A}(t)$ the real and imaginary parts:

$$
\begin{aligned}
|A(t)|= & \frac{|1-\exp (-i t A)|}{t} E_{A}([0, \delta / t])+\frac{|1-\exp (-i t a)|}{t} E_{A}((\delta / t, \infty)) \\
= & \frac{2 \sin (t A / 2)}{t} E_{A}([0, \delta / t])+\frac{2|\sin (t a / 2)|}{t} E_{A}((\delta / t, \infty)), \\
i A(t)= & \frac{1-\cos (t A)}{t} E_{A}([0, \delta / t])+\frac{1-\cos (t a)}{t} E_{A}((\delta / t, \infty)) \\
& +i\left\{\frac{\sin (t A)}{t} E_{A}\left([0, \delta / t]+\frac{\sin (t a)}{t} E_{A}((\delta / t, \infty))\right\}\right. \\
= & G_{A}(t)+i H_{A}(t) .
\end{aligned}
$$

For $0<t<\pi / a(0<t$ if $a=0),|A(t)|, G_{A}(t)$ and $H_{A}(t)$ are all bounded, nonnegative selfadjoint operators on $\mathscr{H}$. For $i B(t)$ we use similar notations $|B(t)|, G_{B}(t)$ and $H_{B}(t)$, replacing $A, a$ by $B, b$, respectively.

It can be easily verified by the spectral theorem and the Lebesgue dominated convergence theorem that, for $T=A$ and $T=B$,

$$
\begin{aligned}
& \lim _{t \downarrow 0} G_{T}(t)^{1 / 2} u=0, \\
& \lim _{t \downarrow 0} H_{T}(t)^{1 / 2} u=\lim _{t \downarrow 0}|T(t)|^{1 / 2} u=T^{1 / 2} u,
\end{aligned}
$$

whenever $u \in D\left[T^{1 / 2}\right]$.

Further define bounded positive selfadjoint operators in $\mathscr{H}$

$$
\begin{gathered}
M(t)=1+H_{A}(t)+H_{B}(t), \\
N(t)=1+|A(t)|+|B(t)|,
\end{gathered}
$$

where $0<t<\min (\pi / a, \pi / b)$. Here and in the following we understand $1 / a=\infty$ (resp. $1 / b=\infty$ ) if $a=0$ (resp. $b=0$ ). We have

$$
\begin{aligned}
S(t) & \equiv 1+i-t^{-1}(F(t)-1) \\
& =1+i(1+A(t)+B(t))+t A(t) B(t) .
\end{aligned}
$$

Set 


$$
\begin{aligned}
S_{0}(t) & =1+i(1+A(t)+B(t)) \\
& =1+G_{A}(t)+G_{B}(t)+i\left(1+H_{A}(t)+H_{B}(t)\right) .
\end{aligned}
$$

To prove (2.4) we first show that

$$
S_{0}(t)^{-1} \underset{s}{\longrightarrow}(1+i+i C)^{-1} P, \quad t \downarrow 0,
$$

and then that

$$
S_{0}(t)^{-1}-S(t)^{-1} \underset{s}{\longrightarrow}, \quad t \downarrow 0 .
$$

I. Proof of (2.4a). Let $f \in \mathscr{H}$. Note that $i A(t)$ and $i B(t)$ are $m$-accretive in $\mathscr{H}$ for $t>0$. Set $u_{t}=S_{0}(t)^{-1} f$. Let $0<t<\min (\pi / a, \pi / b)$. Then $f=S_{0}(t) u_{t}$ and

$$
\begin{aligned}
\left(f, u_{t}\right)= & \left\|u_{t}\right\|^{2}+\left\|G_{A}(t)^{1 / 2} u_{t}\right\|^{2}+\left\|G_{B}(t)^{1 / 2} u_{t}\right\|^{2} \\
& +i\left\{\left\|u_{t}\right\|^{2}+\left\|H_{A}(t)^{1 / 2} u_{t}\right\|^{2}+\left\|H_{B}(t)^{1 / 2} u_{t}\right\|^{2}\right\}
\end{aligned}
$$

It follows that $\left\{u_{t}\right\},\left\{G_{A}(t)^{1 / 2} u_{t}\right\},\left\{G_{B}(t)^{1 / 2} u_{t}\right\},\left\{H_{A}(t)^{1 / 2} u_{t}\right\}$ and $\left\{H_{B}(t)^{1 / 2} u_{t}\right\}$ are all uniformly bounded in $\mathscr{H}$ as $t \downarrow 0$. There exists a subsequence $\left\{t_{n}\right\}_{n=1}^{\infty}$ with $t_{n} \rightarrow 0$ as $n \rightarrow \infty$ along which

$$
\begin{aligned}
& u_{t} \underset{w}{\longrightarrow} u, \quad G_{A}(t)^{1 / 2} u_{t} \underset{w}{\longrightarrow} g_{A}, \quad G_{B}(t)^{1 / 2} u_{t} \longrightarrow w g_{B}, \\
& H_{A}(t)^{1 / 2} u_{t} \underset{w}{\longrightarrow} h_{A}, \quad H_{B}(t)^{1 / 2} u_{t} \underset{w}{\longrightarrow} h_{B} .
\end{aligned}
$$

We first claim that

$$
u \in D\left[C^{1 / 2}\right], \quad g_{A}=g_{B}=0, \quad h_{A}=A^{1 / 2} u, \quad h_{B}=B^{1 / 2} u,
$$

and next that

$$
u \in D[C], \quad P f=(1+i+i C) u \quad \text { or } \quad u=(1+i+i C)^{-1} P f .
$$

In fact, if $v \in D\left[A^{1 / 2}\right]$ then by (2.7) we have

$$
\begin{aligned}
& \left(g_{A}, v\right)=\lim \left(G_{A}(t)^{1 / 2} u_{t}, v\right)=\lim \left(u_{t}, G_{A}(t)^{1 / 2} v\right)=0 \\
& \left(h_{A}, v\right)=\lim \left(H_{A}(t)^{1 / 2} u_{t}, v\right)=\lim \left(u_{t}, H_{A}(t)^{1 / 2} v\right)=\left(u, A^{1 / 2} v\right)
\end{aligned}
$$

the limits taken along $t_{n} \rightarrow 0$. Since $D\left[A^{1 / 2}\right]$ is dense in $\mathscr{H}$, we obtain $g_{A}=0$ and $h_{A} \in D\left[A^{1 / 2}\right], h_{A}=A^{1 / 2} u$. The same is true for $B$. This proves (2.14). To show (2.15) let $v \in D\left[C^{1 / 2}\right]$. Then the right-hand side of

$$
\begin{aligned}
(f, v)= & \left(u_{t}, v\right)+\left(G_{A}(t)^{1 / 2} u_{t}, G_{A}(t)^{1 / 2} v\right)+\left(G_{B}(t)^{1 / 2} u_{t}, G_{B}(t)^{1 / 2} v\right) \\
& +i\left\{\left(u_{t}, v\right)+\left(H_{A}(t)^{1 / 2} u_{t}, H_{A}(t)^{1 / 2} v\right)+\left(H_{B}(t)^{1 / 2} u_{t}, H_{B}(t)^{1 / 2} v\right)\right\}
\end{aligned}
$$

is by (2.7) convergent to 


$$
\begin{aligned}
& (u, v)+i\left\{(u, v)+\left(A^{1 / 2} u, A^{1 / 2} v\right)+\left(B^{1 / 2} u, B^{1 / 2} v\right)\right\} \\
& \quad=(u, v)+i\left\{(u, v)+\left(C^{1 / 2} u, C^{1 / 2} v\right)\right\}
\end{aligned}
$$

along $t_{n} \rightarrow 0$. It follows that $f=(1+i+i C) u$ or $u=0$ according as $f$ belongs to $\mathscr{H}_{0}$ or $\mathscr{H}_{0}^{\perp}$, which yields (2.15).

We have thus seen the weak limits of (2.13) do not depend upon the subsequence chosen. A standard argument concludes that (2.13) holds as $t \downarrow 0$ without taking any subsequence.

Finally we show strong convergence of (2.13). Since the left-hand side of (2.12) converges to

$$
(f, u)=((1+i+i C) u, u)=\|u\|^{2}+i\left\{\|u\|^{2}+\left\|A^{1 / 2} u\right\|^{2}+\left\|B^{1 / 2} u\right\|^{2}\right\},
$$

it follows in view of (2.13) and (2.14) that

$$
\begin{aligned}
& \left\|u_{t}-u\right\|^{2}+\left\|G_{A}(t)^{1 / 2} u_{t}\right\|^{2}+\left\|G_{B}(t)^{1 / 2} u_{t}\right\|^{2} \\
& \quad+i\left\{\left\|u_{t}-u\right\|^{2}+\left\|H_{A}(t)^{1 / 2} u_{t}-A^{1 / 2} u\right\|^{2}+\left\|H_{B}(t)^{1 / 2} u_{t}-B^{1 / 2} u\right\|^{2}\right\}
\end{aligned}
$$

is convergent to 0 . This proves strong convergence, and, in particular, (2.4a).

II. Proof of (2.4b). In order to make the proof transparent we first list some useful facts in the following three lemmas, whose proofs will be given in Section 2.2 .

Let $0<t<\min (\pi / a, \pi / b)$, where $1 / a=\infty$ if $a=0$, and $1 / b=\infty$ if $b=0$.

\section{Lemma 1.}

(i) $\quad\left\|M(t)^{-1 / 2}\right\| \leq 1, \quad\left\|N(t)^{-1 / 2}\right\| \leq 1$ $\mathrm{s}-\lim _{t \downarrow 0} M(t)^{-1 / 2}=\mathrm{s}-\lim _{t \downarrow 0} N(t)^{-1 / 2}=(1+C)^{-1 / 2} P$.

(ii) $\lim _{t \downarrow 0} M(t)^{1 / 2} u=\lim _{t \downarrow 0} N(t)^{1 / 2} u=(1+C)^{1 / 2} u, \quad u \in D\left[C^{1 / 2}\right]$.

(iii) For $0<t<\min (\delta / a, \delta / b)$, $\left\|N(t)^{1 / 2} M(t)^{-1 / 2}\right\| \leq(\cos (\delta / 2))^{-1 / 2}$. $\lim _{t \downarrow 0} N(t)^{1 / 2} M(t)^{-1 / 2} f=f, \quad f \in \mathscr{H}_{0}$.

(iv) $\quad\left\||A(t)|^{1 / 2} N(t)^{-1 / 2}\right\| \leq 1, \quad\left\||B(t)|^{1 / 2} N(t)^{-1 / 2}\right\| \leq 1$. $\lim _{t \downarrow 0}|A(t)|^{1 / 2} N(t)^{-1 / 2} f=A^{1 / 2}(1+C)^{-1 / 2} f, \quad f \in \mathscr{H}_{0}$. $\lim _{t \downarrow 0}|B(t)|^{1 / 2} N(t)^{-1 / 2} f=B^{1 / 2}(1+C)^{-1 / 2} f, \quad f \in \mathscr{H}_{0}$.

\section{Lemma 2.}

$$
\begin{aligned}
& \left\|M(t)^{1 / 2} S_{0}(t)^{-1} M(t)^{1 / 2}\right\| \leq 1 . \\
& \lim _{t \downarrow 0} M(t)^{1 / 2} S_{0}(t)^{-1} M(t)^{1 / 2} f=(1+C)(1+i+i C)^{-1} f, \quad f \in \mathscr{H}_{0} .
\end{aligned}
$$

Lemma 3. Let $0<t<\min (\delta / a, \delta / b)$. 
(i) $\quad\left\|N(t)^{-1 / 2} t A(t) B(t) N(t)^{-1 / 2}\right\| \leq 2 \sin (\delta / 2)$.

$$
\left\|N(t)^{1 / 2} S(t)^{-1} N(t)^{1 / 2}\right\| \leq(\cos (\delta / 2)-\sin (\delta / 2))^{-1} .
$$

(ii) $\lim _{t \downarrow 0} N(t)^{-1 / 2} t A(t) B(t) N(t)^{-1 / 2} f=0, f \in \mathscr{H}_{0}$.

Now we turn to the proof of (2.4b). Observe

$$
\begin{aligned}
& S_{0}(t)^{-1}-S(t)^{-1} \\
&= S(t)^{-1} t A(t) B(t) S_{0}(t)^{-1} \\
&= N(t)^{-1 / 2}\left(N(t)^{1 / 2} S(t)^{-1} N(t)^{1 / 2}\right)\left(N(t)^{-1 / 2} t A(t) B(t) N(t)^{-1 / 2}\right) \\
& \cdot\left(N(t)^{1 / 2} M(t)^{-1 / 2}\right)\left(M(t)^{1 / 2} S_{0}(t)^{-1} M(t)^{1 / 2}\right) M(t)^{-1 / 2}
\end{aligned}
$$

By Lemmas 1-3 all the six factors in the last formula above are uniformly bounded as operators on $\mathscr{H}$. As $t \downarrow 0$, the third, fourth and fifth factors converge strongly on $\mathscr{H}_{0}$ to 0,1 and $(1+C)(1+i+i C)^{-1}$, respectively, while the last factor converges strongly on $\mathscr{H}$ to $(1+C)^{-1 / 2} P$, by Lemma 1 . This proves (2.4b), completing the proof of Theorem 1 .

\subsection{Proofs of Lemmas 1-3.}

Proof of Lemma 1. (i) The assertions can be shown by a similar argument used in the proof of $(2.4 \mathrm{a})$ in Section 2.1. In fact, for $f \in \mathscr{H}$ consider instead of (2.12)

$$
\begin{aligned}
& \left(f, u_{t}\right)=\left\|u_{t}\right\|^{2}+\left\|H_{A}(t)^{1 / 2} u_{t}\right\|^{2}+\left\|H_{B}(t)^{1 / 2} u_{t}\right\|^{2}, \quad u_{t}=M(t)^{-1} f, \\
& \left(f, v_{t}\right)=\left\|v_{t}\right\|^{2}+\left\||A(t)|^{1 / 2} v_{t}\right\|^{2}+\left\||B(t)|^{1 / 2} v_{t}\right\|^{2}, \quad v_{t}=N(t)^{-1} f .
\end{aligned}
$$

(ii) We give the proof only for $M(t)$. For $N(t)$ the proof is similar. Let $u \in D\left[C^{1 / 2}\right]$. In view of (2.1) and (2.7),

$$
\left\|M(t)^{1 / 2} u\right\|^{2}=\|u\|^{2}+\left\|H_{A}(t)^{1 / 2} u\right\|^{2}+\left\|H_{B}(t)^{1 / 2} u\right\|^{2}
$$

converges to $\|u\|^{2}+\left\|C^{1 / 2} u\right\|^{2}=\left\|(1+C)^{1 / 2} u\right\|^{2}$ as $t \downarrow 0$. It remains to show $M(t)^{1 / 2} u \underset{w}{\longrightarrow}(1+C)^{1 / 2} u$. By boundedness of $\left\{M(t)^{1 / 2} u\right\}$ there exists a subsequence $\left\{t_{n}\right\}_{n=1}^{\infty}$, with $t_{n} \rightarrow 0$, along which $M(t)^{1 / 2} u \overleftrightarrow{w} g$. We have

$$
\|g\| \leq \lim \inf \left\|M(t)^{1 / 2} u\right\|=\lim \left\|M(t)^{1 / 2} u\right\|=\left\|(1+C)^{1 / 2} u\right\|,
$$

and by Lemma 1 (i) for $v \in \mathscr{H}$

$$
(u, v)=\lim \left(M(t)^{1 / 2} u, M(t)^{-1 / 2} v\right)=\left(g,(1+C)^{-1 / 2} P v\right),
$$

the limit taken along $t_{n} \rightarrow 0$. It follows that $g=(1+C)^{1 / 2} u$. Thus the limit is independent of the subsequence chosen. A standard argument shows weak convergence. 
(iii) Let $0<t<\min (\delta / a, \delta / b)$. We have for $g \in \mathscr{H}$

$$
\begin{aligned}
& (|A(t)| g, g) \\
= & \left(\frac{2 \sin (t A / 2)}{t} E_{A}([0, \delta / t]) g, E_{A}([0, \delta / t]) g\right) \\
& +\frac{2 \sin (t a / 2)}{t}\left\|E_{A}((\delta / t, \infty)) g\right\|^{2} \\
\leq & (\cos (\delta / 2))^{-1}\left\{\left(\frac{\sin (t A)}{t} E_{A}([0, \delta / t]) g, E_{A}([0, \delta / t]) g\right)\right. \\
& \left.+\frac{\sin (t a)}{t}\left\|E_{A}((\delta / t, \infty)) g\right\|^{2}\right\} \\
= & (\cos (\delta / 2))^{-1}\left(H_{A}(t) g, g\right),
\end{aligned}
$$

and the same is true for $|B(t)|$ and $H_{B}(t)$. It follows that

$$
\begin{aligned}
\left\|N(t)^{1 / 2} M(t)^{-1 / 2} g\right\|^{2} & =\left((1+|A(t)|+|B(t)|) M(t)^{-1 / 2} g, M(t)^{-1 / 2} g\right) \\
& \leq(\cos (\delta / 2))^{-1}\left(\left(1+H_{A}(t)+H_{B}(t)\right) M(t)^{-1 / 2} g, M(t)^{-1 / 2} g\right) \\
& =(\cos (\delta / 2))^{-1}\|g\|^{2},
\end{aligned}
$$

whence follows the first assertion.

Next we show the second one. Now that $N(t)^{1 / 2} M(t)^{-1 / 2}$ is uniformly bounded as operators on $\mathscr{H}$, we have only to show that for $f \in \mathscr{H}_{0}, M(t)^{1 / 2}$. $N(t)^{-1 / 2} f \rightarrow f$. Moreover, since $\left\|M(t)^{1 / 2} N(t)^{-1 / 2}\right\| \leq 1$, it suffices to show that $M(t)^{1 / 2} N(t)^{-1 / 2} f \underset{w}{\longrightarrow}$. To see it, let $f_{t}=M(t)^{1 / 2} N(t)^{-1 / 2} f, f \in \mathscr{H}_{0}$. Then $\left\|f_{t}\right\| \leq$ $\|f\|$, and so there is a subsequence $\left\{t_{n}\right\}_{n=1}^{\infty}, t_{n} \rightarrow 0$, along which $f_{t} \longrightarrow f_{0}$. A similar argument used in the proof of Lemma 1 (ii) with Lemma 1 (i) and (ii) can conclude that $f_{0}=f$ and $f_{t} \vec{w} f$ as $t \downarrow 0$, without taking any subsequence.

(iv) If $f \in \mathscr{H}$,

$$
\|f\|^{2}=\left\|N(t)^{-1 / 2} f\right\|^{2}+\left\||A(t)|^{1 / 2} N(t)^{-1 / 2} f\right\|^{2}+\left\||B(t)|^{1 / 2} N(t)^{-1 / 2} f\right\|^{2} .
$$

Hence

$$
\left\||A(t)|^{1 / 2} N(t)^{-1 / 2} f\right\| \leq\|f\|, \quad\left\||B(t)|^{1 / 2} N(t)^{-1 / 2} f\right\| \leq\|f\| .
$$

Now let $f \in \mathscr{H}_{0}$. By Lemma 1 (i), $N(t)^{-1 / 2} f \rightarrow(1+C)^{-1 / 2} f$. There is a subsequence $\left\{t_{n}\right\}_{n=1}^{\infty}, t_{n} \rightarrow 0$, along which

$$
|A(t)|^{1 / 2} N(t)^{-1 / 2} f \underset{w}{\longrightarrow} f_{A}, \quad|B(t)|^{1 / 2} N(t)^{-1 / 2} f \underset{w}{\longrightarrow} f_{B} .
$$

Analogously to the proof of (2.4a) in Section 2.1 we have $f_{A}=A^{1 / 2}(1+C)^{-1 / 2} f$, $f_{B}=B^{1 / 2}(1+C)^{-1 / 2} f$, and $(2.17)$ holds as $t \downarrow 0$, without taking any subsequence.

It remains to show strong convergence. To this end it suffices to establish

$$
\lim \sup _{t \downarrow 0}\left\||A(t)|^{1 / 2} N(t)^{-1 / 2} f\right\| \leq\left\|A^{1 / 2}(1+C)^{-1 / 2} f\right\|,
$$




$$
\lim \sup _{t \downarrow 0}\left\||B(t)|^{1 / 2} N(t)^{-1 / 2} f\right\| \leq\left\|B^{1 / 2}(1+C)^{-1 / 2} f\right\| .
$$

Suppose there is an $f$ in $\mathscr{H}_{0}$ such that at least one of (2.18) and (2.19), say (2.18), does not hold. Then it should follow that

$$
\begin{aligned}
& \left\|C^{1 / 2}(1+C)^{-1 / 2} f\right\|^{2} \\
& \quad=\left\|A^{1 / 2}(1+C)^{-1 / 2} f\right\|^{2}+\left\|B^{1 / 2}(1+C)^{-1 / 2} f\right\|^{2} \\
& \quad<\lim \sup \left\||A(t)|^{1 / 2} N(t)^{-1 / 2} f\right\|^{2}+\liminf \left\||B(t)|^{1 / 2} N(t)^{-1 / 2} f\right\|^{2} \\
& \quad \leq \lim \sup \left\{\left\||A(t)|^{1 / 2} N(t)^{-1 / 2} f\right\|^{2}+\left\||B(t)|^{1 / 2} N(t)^{-1 / 2} f\right\|^{2}\right\} \\
& \quad=\lim \left\{\|f\|^{2}-\left\|N(t)^{-1 / 2} f\right\|^{2}\right\} \\
& \quad=\|f\|^{2}-\left\|(1+C)^{-1 / 2} f\right\|^{2} \\
& \quad=\left\|C^{1 / 2}(1+C)^{-1 / 2} f\right\|^{2},
\end{aligned}
$$

which is a contradiction. Here we have used (2.16) and Lemma 1 (i). This ends the proof of Lemma 1.

Proof of Lemma 2. Let $f \in \mathscr{H}$. Set for $t>0$

$$
u_{t}=M(t)^{1 / 2} S_{0}(t)^{-1} M(t)^{1 / 2} f \text { or } f=M(t)^{-1 / 2} S_{0}(t) M(t)^{-1 / 2} u_{t} .
$$

Note $M(t)^{1 / 2} S_{0}(t)^{-1} M(t)^{1 / 2}$ is a bounded linear operator of $\mathscr{H}$ onto itself. Then

$$
\begin{aligned}
\left(f, u_{t}\right) & =\left(S_{0}(t) M(t)^{-1 / 2} u_{t}, M(t)^{-1 / 2} u_{t}\right) \\
& =\left(\left(1+G_{A}(t)+G_{B}(t)\right) M(t)^{-1 / 2} u_{t}, M(t)^{-1 / 2} u_{t}\right)+i\left(u_{t}, u_{t}\right) .
\end{aligned}
$$

Since the first term of the last member of $(2.20)$ is real and positive, we obtain for $g \in \mathscr{H}$

$$
\left|\left(M(t)^{-1 / 2} S_{0}(t) M(t)^{-1 / 2} g, g\right)\right| \geq\|g\|^{2},
$$

whence follows the first assertion.

To show the second one, let $f \in D\left[C^{1 / 2}\right]$. By boundedness of $\left\{u_{t}\right\}$ there is a subsequence $\left\{t_{n}\right\}_{n=1}^{\infty}, t_{n} \rightarrow 0$, along which $u_{t} \vec{w} u_{0}$. We have for $v \in D\left[C^{1 / 2}\right]$

$$
\begin{aligned}
\left(u_{0}, v\right) & =\lim _{t \downarrow 0}\left(M(t)^{1 / 2} S_{0}(t)^{-1} M(t)^{1 / 2} f, v\right) \\
& =\lim _{t \downarrow 0}\left(S_{0}(t)^{-1} M(t)^{1 / 2} f, M(t)^{1 / 2} v\right) \\
& =\left((1+i+i C)^{-1}(1+C)^{1 / 2} f,(1+C)^{1 / 2} v\right) \\
& =\left((1+C)(1+i+i C)^{-1} f, v\right) .
\end{aligned}
$$

Here we have used Lemma 1 (ii) and (2.4a). It follows that $P u_{0}=(1+C)$. $(1+i+i C)^{-1} f$. This is also valid for $f \in \mathscr{H}_{0}$, since $D\left[C^{1 / 2}\right]$ is dense in $\mathscr{H}_{0}$ and $M(t)^{1 / 2} S_{0}(t)^{-1} M(t)^{1 / 2}$ is uniformly bounded as operators on $\mathscr{H}$. Now (2.20) with $f \in \mathscr{H}_{0}$ yields 


$$
\begin{aligned}
\lim \sup \left\|u_{t}\right\|^{2} & =\lim \sup \operatorname{Im}\left(f, u_{t}\right)=\lim \operatorname{Im}\left(f, u_{t}\right) \\
& =\operatorname{Im}\left(f,(1+C)(1+i+i C)^{-1} f\right)=\left\|(1+C)(1+i+i C)^{-1} f\right\|^{2} .
\end{aligned}
$$

Hence $u_{0}=P u_{0}$ or $u_{0}=(1+C)(1+i+i C)^{-1} f$. Thus the limit does not depend upon the subsequence chosen. By the same argument as before, $u_{t}$ converges strongly to $(1+C)(1+i+i C)^{-1} f$ as $t \downarrow 0$, without taking any subsequence. This completes the proof of Lemma 2.

Proof of Lemma 3. (i) First note the polar decompositions of the normal operators $A(t)$ and $B(t)$ :

$$
A(t)=U(t)|A(t)|=|A(t)| U(t), \quad B(t)=V(t)|B(t)|=|B(t)| V(t),
$$

where $U(t)$ and $V(t)$ are partial isometries on $\mathscr{H}$. We claim that

$$
\left\|t^{1 / 2}|A(t)|^{1 / 2} U(t) V(t) t^{1 / 2}|B(t)|^{1 / 2}\right\| \leq 2 \sin (\delta / 2),
$$

for $0<t<\min (\delta / a, \delta / b)$. To see it we have only to show

$$
\begin{aligned}
& \left\|t^{1 / 2}|A(t)|^{1 / 2}\right\| \leq(2 \sin (\delta / 2))^{1 / 2} \\
& \left\|t^{1 / 2}|B(t)|^{1 / 2}\right\| \leq(2 \sin (\delta / 2))^{1 / 2}
\end{aligned}
$$

But this can be easily verified by definitions of $|A(t)|$ and $|B(t)|$. It follows in view of (2.22) that, for $g \in \mathscr{H}$,

$$
\begin{aligned}
\mid( & \left.N(t)^{-1 / 2} t A(t) B(t) N(t)^{-1 / 2} g, g\right) \mid \\
\leq & \mid\left(\left(t^{1 / 2}|A(t)|^{1 / 2} U(t) V(t) t^{1 / 2}|B(t)|^{1 / 2}\right)\right. \\
& \left.\cdot\left(|B(t)|^{1 / 2} N(t)^{-1 / 2}\right) g,\left(|A(t)|^{1 / 2} N(t)^{-1 / 2}\right) g\right) \mid \\
\leq & 2 \sin (\delta / 2)\left\||B(t)|^{1 / 2} N(t)^{-1 / 2} g\right\|\left\||A(t)|^{1 / 2} N(t)^{-1 / 2} g\right\| \\
\leq & \sin (\delta / 2)\left\{\left\||B(t)|^{1 / 2} N(t)^{-1 / 2} g\right\|^{2}+\left\||A(t)|^{1 / 2} N(t)^{-1 / 2} g\right\|^{2}\right\} \\
& =\sin (\delta / 2)\left((|A(t)|+|B(t)|) N(t)^{-1 / 2} g, N(t)^{-1 / 2} g\right) \\
\leq & \sin (\delta / 2)\|g\|^{2} .
\end{aligned}
$$

This implies the first assertion.

To show the second one, let $g \in \mathscr{H}$ again. By (2.21) and Lemma 1 (iii)

$$
\begin{aligned}
& \left|\left(N(t)^{-1 / 2} S_{0}(t) N(t)^{-1 / 2} g, g\right)\right| \\
& \quad=\left|\left(M(t)^{-1 / 2} S_{0}(t) M(t)^{-1 / 2}\left(M(t)^{1 / 2} N(t)^{-1 / 2}\right) g,\left(M(t)^{1 / 2} N(t)^{-1 / 2}\right) g\right)\right| \\
& \quad \geq\left\|M(t)^{1 / 2} N(t)^{-1 / 2} g\right\|^{2} \\
& \quad \geq \cos (\delta / 2)\|g\|^{2} .
\end{aligned}
$$

It follows together with (2.24) that 


$$
\begin{aligned}
& \left|\left(N(t)^{-1 / 2} S(t) N(t)^{-1 / 2} g, g\right)\right| \\
& \quad \geq\left|\left(N(t)^{-1 / 2} S_{0}(t) N(t)^{-1 / 2} g, g\right)\right|-\left|\left(N(t)^{-1 / 2} t A(t) B(t) N(t)^{-1 / 2} g, g\right)\right| \\
& \quad \geq(\cos (\delta / 2)-\sin (\delta / 2))\|g\|^{2} .
\end{aligned}
$$

This yields the second assertion.

(ii) Observe

$$
\begin{aligned}
& N(t)^{-1 / 2} t A(t) B(t) N(t)^{-1 / 2} \\
= & \left(N(t)^{-1 / 2}|A(t)|^{1 / 2}\right)\left(t^{1 / 2}|A(t)|^{1 / 2} U(t) V(t)\right)\left(t^{1 / 2}|B(t)|^{1 / 2}\right)\left(|B(t)|^{1 / 2} N(t)^{-1 / 2}\right) .
\end{aligned}
$$

All the four factors above are uniformly bounded as operators on $\mathscr{H}$, by Lemma 1 (iv) and (2.23). By the spectral theorem and Lemma 1 (iv) again, the third factor converges strongly on $\mathscr{H}$ to 0 and the last one strongly on $\mathscr{H}_{0}$ to $B^{1 / 2}(1+C)^{-1 / 2}$. This proves the assertion, concluding the proof of Lemma 3.

\section{§3. A General Class of Product Formulas}

In this section we shall indicate a more general class of product formulas including (1.3).

For $0<\tau \leq \infty, 0<\mu<v \leq 1, \gamma \in \boldsymbol{R}$ and $\varepsilon= \pm 1, \mathscr{F}_{v, \mu}(\tau, \gamma, \varepsilon)$ denotes the class of the bounded, complex-valued functions $\zeta(t ; \lambda)$ on $[0, \tau) \times[\gamma, \infty)$ satisfying the following conditions:

(i) for each fixed $t, \zeta(t ; \lambda)$ is Borel measurable in $\lambda$;

(ii) for each fixed $\lambda, \zeta(t ; \lambda)$ is continuous in $t$ with

$$
\zeta(0 ; \lambda)=1, \quad(\partial / \partial t) \zeta(0 ; \lambda)=i \varepsilon \lambda ;
$$

(iii) there exists a positive constant $K$ such that

$$
|1-\zeta(t ; \lambda)| \leq K t(1+|\lambda|), \quad \text { for all }(t, \lambda) \in[0, \tau) \times[\gamma, \infty) ;
$$

(iv) for all $(t, \lambda)$ in $[0, \tau) \times[\gamma, \infty)$,

$$
\begin{aligned}
& \operatorname{Re}\left(\zeta(t ; \lambda) e^{-i \varepsilon t \gamma}\right) \leq 1, \quad \varepsilon \operatorname{Im}\left(\zeta(t ; \lambda) e^{-i \varepsilon t \gamma}\right) \geq 0, \\
& \left|1-\zeta(t ; \lambda) e^{-i \varepsilon t \gamma}\right| \leq \min \left\{2 \mu, v^{-1}\left|\operatorname{Im}\left(\zeta(t ; \lambda) e^{-i \varepsilon t \gamma}\right)\right|\right\} .
\end{aligned}
$$

Examples. For $0<\delta<\pi / 2, c \geq \gamma$ and $\varepsilon= \pm 1$, the following functions $\zeta_{\gamma, c}(t ; \lambda)$ and $\zeta_{\gamma, c}^{\prime}(t ; \lambda)$ belong to $\mathscr{F}_{v, \mu}(\delta /(c-\gamma), \gamma, \varepsilon)$ with $v=\cos (\delta / 2)$ and $\mu=\sin (\delta / 2)$ :

$$
\begin{aligned}
\zeta_{\gamma, c}(t ; \lambda) & =e^{i \varepsilon t \lambda} \chi_{[\gamma, \gamma+\delta / t]}(\lambda)+e^{i \varepsilon t c} \chi_{(\gamma+\delta / t, \infty)}(\lambda) \\
& =e^{i \varepsilon t \lambda} \chi_{[0, \delta]}(t(\lambda-\gamma))+e^{i \varepsilon t c} \chi_{(\delta, \infty)}(t(\lambda-\gamma)),
\end{aligned}
$$




$$
\zeta_{\gamma, c}^{\prime}(t ; \lambda)=e^{i \varepsilon t \lambda} \rho_{[0, \delta]}(t(\lambda-\gamma))+e^{i \varepsilon t c}\left\{1-\rho_{[0, \delta]}(t(\lambda-\gamma))\right\} .
$$

Here $\chi_{I}(\lambda)$ is the indicator function of a Borel set $I$ in $\mathbb{R}$, and $\rho_{[0, \delta]}(\lambda)$ is a realvalued $C^{\infty}$ function in $\mathbb{R}, 0 \leq \rho_{[0, \delta]}(\lambda) \leq 1$, such that

$$
\rho_{[0, \delta]}(\lambda)=1, \quad 0 \leq \lambda \leq 3 \delta / 4, \text { and }=0, \lambda \geq \delta \text {. }
$$

The following theorem extends Theorem 1 .

Theorem 3. Let $A$ and $B$ be the same as in Section 1.1. Let $\varepsilon= \pm 1$. For some $0<\tau \leq \infty$ and $0<\mu<\nu \leq 1$, let $\alpha \in \mathscr{F}_{v, \mu}\left(\tau, \gamma_{A}, \varepsilon\right)$ and $\beta \in \mathscr{F}_{v, \mu}\left(\tau, \gamma_{B}, \varepsilon\right)$, and assume that

$$
\sup _{\lambda \geq \gamma_{A}}|\alpha(t ; \lambda)| \cdot \sup _{\lambda \geq \gamma_{B}}|\beta(t ; \lambda)| \leq 1, \quad \text { for } t \in[0, \tau) \text {. }
$$

Then for $f \in \mathscr{H}_{0}=P \mathscr{H}$,

$$
\{\alpha(t / n ; A) \beta(t / n ; B)\}^{n} f \rightarrow e^{i \varepsilon t(A+B)} f, \quad n \rightarrow \infty,
$$

uniformly in bounded intervals in $t \geq 0$.

Proof. We have only to treat the case $\varepsilon=-1$ and $\gamma_{A}=\gamma_{B}=0$, so that both $\alpha$ and $\beta$ belong to $\mathscr{F}_{v, \mu}(\tau, 0,-1)$. Define $F(t)=\alpha(t ; A) \beta(t ; B), t \geq 0$, and $A(t)=(i t)^{-1}\{1-\alpha(t ; A)\}, B(t)=(i t)^{-1}\{1-\beta(t ; B)\}, t>0$. The remaining proof proceeds just in the same way as that of Theorem 1 in Section 2.

\section{Remarks}

1. Theorem 3 is valid with the functions (3.1) and (3.2) in Examples preceding Theorem 3 for $\alpha(t ; \lambda)$ and $\beta(t ; \lambda)$. The case in which $\alpha$ and $\beta$ are the functions $\zeta_{\gamma, c}$ with $(\gamma, c)=\left(\gamma_{A}, a\right)$ and $(\gamma, c)=\left(\gamma_{B}, b\right)$ respectively is nothing but Theorem 1.

2. Application of the case in which $\alpha$ and $\beta$ are the functions $\zeta_{\gamma, c}^{\prime}$ with $(\gamma, c)=\left(\gamma_{A}, a\right)$ and $(\gamma, c)=\left(\gamma_{B}, b\right)$ respectively to the Schrödinger equation $(0.3)$ yields the formula (1.6) in Theorem 2 with the following different cutoff functions:

$$
\begin{array}{ll}
\Xi(t ; \xi)=\rho_{[0, \delta]}\left(t \xi^{2} / 2\right), & X(t ; x)=\rho_{[0, \delta]}(t(V(x)-\gamma)), \\
\Xi^{\prime}(t ; \xi)=1-\Xi(t ; \xi), & X^{\prime}(t ; x)=1-X(t ; x) .
\end{array}
$$

Here notice that $\Xi(t ; \xi)$ and $X(t ; x)$ are regular, respectively, everywhere and where $V(x)$ is regular. 


\section{References}

[1] Chernoff, P. R., Product Formulas, Nonlinear Semigroups, and Addition of Unbounded Operators, Memoirs of Amer. Math. Soc., 140, 1974.

[2] Chorin, A. J., Hughes, T. J. R., McCracken, M. F. and Marsden, J. E., Product formulas and numerical algorithms, Commun. Pure Appl. Math., 31 (1978), 205-256.

[3] Faris, W. G., The product formula for semigroups defined by Friedrichs extensions, Pacific J. Math., 22 (1967), 47-70.

[4] Garrod, C., Hamiltonian path-integral methods, Rev. Mod. Phys., 38 (1966), 483-494.

[5] Kato, T., Perturbation Theory for Linear Operators, Springer, Berlin-HeidelbergNew York 1966; 2nd ed., 1976.

[6] — Schrödinger operators with singular potentials, Israel J. Math., 13 (1972), $135-148$.

[ 7 ] — On the Trotter-Lie product formula, Proc. Japan Acad., 50 (1974), 694-698.

[8] — Trotter's product formula for an arbitrary pair of selfadjoint contraction semigroups, Topics in Functional Analysis, Essays dedicated to M. G. Krein on the occasion of his 70th birthday, Advances in Mathematics Supplementary Studies, 3, Academic Press, New York, 1978, 185-195.

[9] Kato, T. and Masuda, K., Trotter's product formula for nonlinear semigroups generated by the subdifferentials of convex functionals, J. Math. Soc. Japan, 30 (1978), 169-178.

[10] Mizrahi, M. M., Phase space path integrals, without limiting procedure, J. Math. Phys., 19 (1978), 298-307.

[11] Nelson, E., Feynman integrals and the Schrödinger equation, J. Math. Phys., 5 (1964), 332-343.

[12] Reed, M. and Simon, B., Methods of Modern Mathematical Physics, I, Functional Analysis, II, Fourier Analysis, Self-Adjointness, Academic Press, New York, 1972, 1975.

[13] Simon, B., Quantum Mechanics for Hamiltonians Defined as Quadratic Forms, Princeton Series in Physics, Princeton Univ. Press, Princeton, 1971. 